\title{
Coverage, Capacity and Interference Analysis for an Aerial Base Station in Different Environments
}

\author{
Daniele Giovanni Cileo, Navuday Sharma, Maurizio Magarini \\ Dipartimento di Elettronica, Informazione e Bioingegneria \\ Politecnico di Milano, Milan, Italy \\ daniele.cileo@mail.polimi.it, navuday.sharma@polimi.it, maurizio.magarini@polimi.it
}

\begin{abstract}
With the advancing $5 \mathrm{G}$ technology of base stations mounted on aerial platforms, such as unmanned aerial vehicles, the issue of coverage area, capacity and inter-cell interference is assuming higher importance for the cellular networks. In this paper, we follow a deterministic approach to analyze these problems using the data obtained using a commercial software for wireless electromagnetic wave propagation. We analyze the above mentioned parameters by varying the threshold of the received power. Also, we find an optimal altitude and power consumption model for an aerial base station. Simulations were carried out in three generalized environments, Suburban, Urban and Urban High Rise, developed according to ITU-R parameters. To derive these results we used an air-to-ground channel model obtained from the analysis of simulation data.
\end{abstract}

Index Terms-Cell Coverage, Aerial Base Station, Air-toGround Channel, Ray Tracing, SINR analysis, Power Consumption Model, Optimal Altitude, LAP

\section{INTRODUCTION}

Unmanned Aerial Vehicles (UAVs), commonly known as drones have been used as a Low Altitude Platforms (LAPs) for many purposes. Initially, they were massively used for military purposes, like surveillance or reconnaissance activities [1], [2]. Nowadays, they have received enormous interest in civil applications too, like search and rescue, weather detection, wildlife monitoring, farming, film making etc [3]. Recently, with the development of 5G technology, the idea of using base stations mounted UAVs has gained substantial interest in research literature [4], [5], with the aim of getting an increment in cellular networks capacity and coverage area. This is also what is addressed in this paper.

We consider an Aerial Base Station (ABS), on-boards UAV, that provides cellular network to a mobile Ground Station (GS) using an appropriate air-to-ground (A2G) channel model, which has been obtained using the deterministic solution of ray tracing. Such a system is practically advantageous for events where large number of users in a small area access high speed data simultaneously like, for example, concerts, mass rallies, sport, and cultural events. Such architecture has been previously proposed for rescue operations in case of disasters, where terrestrial cellular networks become non-functional [6].

Some previous work is available making similar analysis. In [7], [8] authors provide the analytical solutions for coverage and comparison of sum rate and power gains of ABS with terrestrial base station using a generic channel model. However, they do not take into account different generalized environ- ments defining some fundamental characteristics such as, for example, Path Loss Exponent (PLE) or shadowing. In these references numerical results have not been verified neither using real measurements nor by accurate channel models like those provided by ray tracing or any other deterministic tool. Similarly, in [9] a formulation is provided for Line of Sight (LOS) probability and optimal altitude but without taking into account large and small scale fading effects. In this paper we take into account all these limitations to provide more accurate results. Different types of generalized environments will be considered in order to get more realistic results. We also characterize $\mathrm{A} 2 \mathrm{G}$ channel for these environments and take into account large and small scale fading effects in order to maintain high accuracy.

This paper is organized as follows. Section II describes the simulation setup used to perform ray tracing simulations. In Sec. III we discuss about the cell coverage calculation and optimal ABS height. The power consumption model of the ABS and its dependency on transmitted power and power needed by the ABS/drone to fly are reported in Sec. IV. In Sec. V, A2G channel model is shown, mentioning the large and small scale fading effects. Section VI reports the formulation of Signal to Noise (SNR) and Capacity, whereas Sec. VII reports about Signal to interference (SIR) and Signal to interference plus Noise (SINR) analysis. Finally, Sec. VIII provides the results and discussion for above analysis with the conclusion drawn in Sec. IX.

\section{Simulation Setup}

In order to perform simulations we considered three different environments: Suburban, Urban, and Urban High Rise. These environments, shown in Fig.1, were created using 3ds Max computer-aided-design software [10], based on ITU$\mathrm{R}$ parameters [9]. According to ITU-R specifications, the buildings were uniformly distributed over the environment with their height following a Rayleigh distribution. It is worth noting that the generalization of these environments gives us the benefit to approximate the results for any city around the world. The environment size was kept to be $2000 \times 2000 \mathrm{~m}^{2}$. However, to reduce the computation time, the simulations were performed only on an area of $1000 \times 1000 \mathrm{~m}^{2}$. Two snapshots from each environment were created to verify and improve the accuracy of results. A number of 32.500 receivers (Rxs), the red dots in Fig.1, were placed uniformly on the streets in 

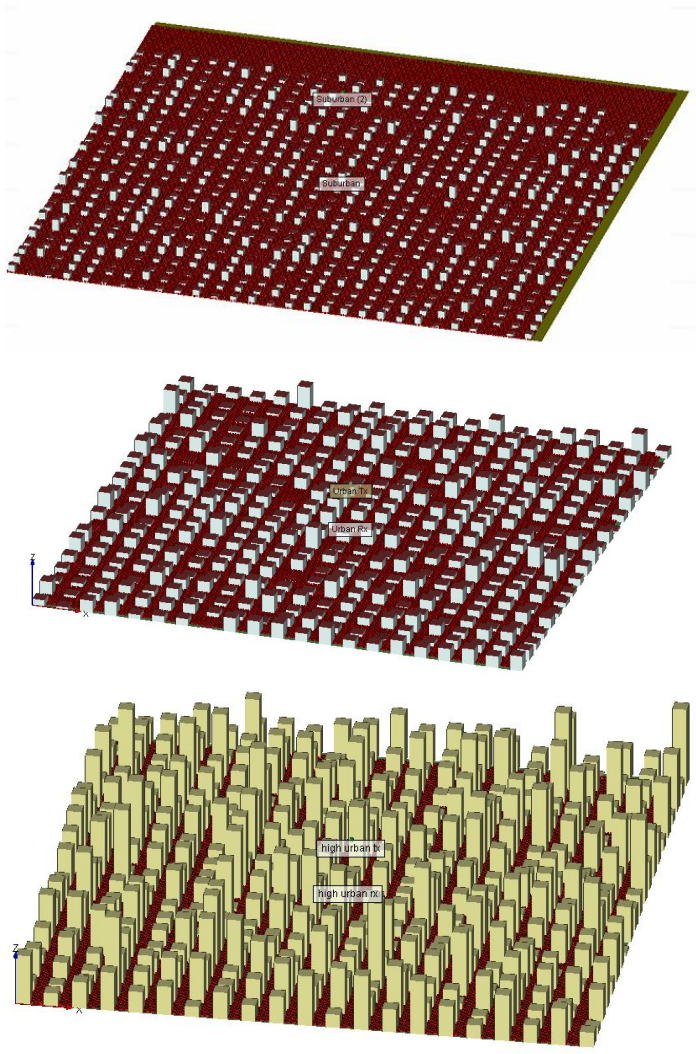

Figure 1. Measurement scenarios for ray tracing simulations.

the city spaced $5 \mathrm{~m}$ apart from each other and $2 \mathrm{~m}$ above the ground. The transmitter (Tx) was placed at the center of the snapshot. All the Rxs and the Tx were equipped with isotropic antennas, that is, ideal configuration to get general results. The $\mathrm{Rx}$ threshold was varied to analyze the coverage area behavior. A noise figure of $3 \mathrm{~dB}$ was set in the simulator. The simulations were performed with Tx heights from $100 \mathrm{~m}$ to $2000 \mathrm{~m}$ with interval for every $100 \mathrm{~m}$, in order to maintain the sensitivity of results. Another set of simulations were carried at a fixed optimal height obtained for ABS and varying the Tx power from $18 \mathrm{dBm}$ to $46 \mathrm{dBm}$. A sinusoid waveform was transmitted at $2.4 \mathrm{GHz}$ carrier frequency and $20 \mathrm{MHz}$ signal bandwidth, keeping the standard sea level atmospheric conditions. The simulations were performed in Wireless InSite 3.0.1 environment [11], whose accuracy, compared to practically measured results, is well defined in [12].

\section{Optimal height of Aerial Base Station for MAXIMUM COVERAGE}

Low altitude platforms are quasi-stationary aerial bodies, such as rotor-crafts, balloons, helikites, drones etc. In this paper, we consider our LAP as a drone because of easy maneuverability, reliability and better stability in wind conditions. For the coverage analysis, with respect to wireless communications, we consider it to be defined as the portion of the cell in which the received power of GS is above

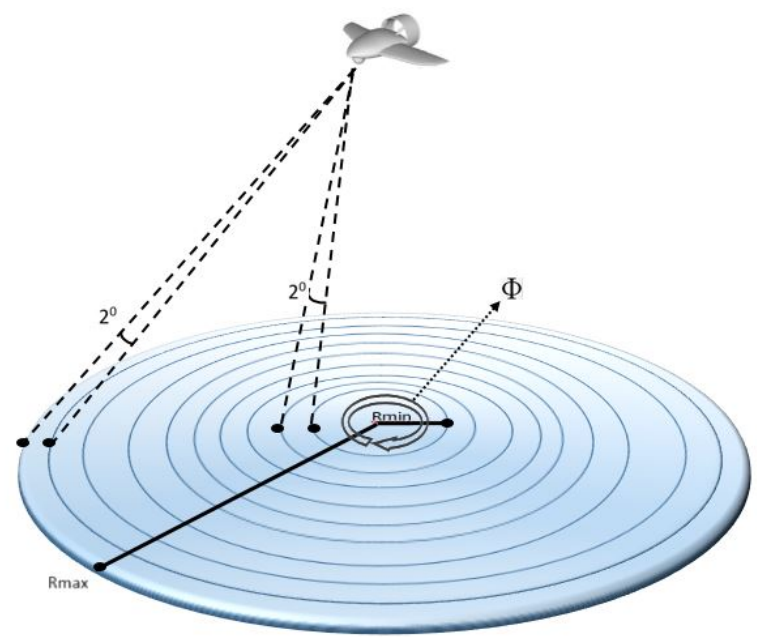

Figure 2. Sectorization used for coverage analysis from ABS.

Table I

Cell Area for the simulation

\begin{tabular}{|l|l|l|l|l|}
\hline Scenario & $\begin{array}{l}\text { Cell Area } \\
\left(\mathrm{m}^{2}\right)\end{array}$ & $\begin{array}{l}\text { No. } \\
\text { of } \\
\text { build- } \\
\text { ings }\end{array}$ & $\begin{array}{l}\text { Building } \\
\text { Area } \\
\left(\mathrm{m}^{2}\right)\end{array}$ & $\begin{array}{l}\text { Final } \\
\text { Cell } \\
\text { Area } \\
\left(\mathrm{m}^{2}\right)\end{array}$ \\
\hline Suburban & $\simeq 104.000$ & 750 & 10.379 & 93.543 \\
\hline Urban & $\simeq 104.000$ & 500 & 31.189 & 72.733 \\
\hline Urban High Rise & $\simeq 104.000$ & 300 & 51.961 & 51.961 \\
\hline
\end{tabular}

the threshold, which is established by the mobile operator. Therefore, following [13], the cell coverage is defined by

$$
C=\frac{1}{A_{c}} \int_{A_{C}} r \cdot P\left(P_{r x}(r) \geq P_{\min }\right) d r d \phi,
$$

where $A_{C}$ is the cell area of chosen radius $200 \mathrm{~m}, r$ is the distance between $\mathrm{Rx}$ and $\mathrm{ABS}$, and $P$ is the probability that the received power $P_{r x}$ is greater than the threshold $P_{\min }$. For obtaining the coverage, a sectorization of the snapshot was done on the basis of $2^{\circ}$ elevation angle between the ABS and GS as shown in Fig. 2. The purpose of sectorization was only to discretize the simulation area for improving accuracy of the results while implementing (1). For each sector, the probability $P$ was calculated from the simulated data for the Rxs within that sector at a given distance from the Tx. The cell area was taken to be of radius $200 \mathrm{~m}$ by removing the building area as shown in Table I.

\section{Optimal Power Consumption of Aerial Base STATION FOR MAXIMUM COVERAGE}

One of the most important aspect when using ABSs is the amount of power they are consuming while flying. For this reason here we use a power consumption model that takes into account both the power needed by the drone to hover $P_{\text {drone }}$, i.e., the power needed for flying, assuming $100 \%$ efficiency 
[14], and the transmitted power $P_{T X}$ from the ABS. The power needed by the drone is given by

$$
P_{\text {drone }}=\sqrt{\frac{(2 M g)^{3}}{16 \rho A}}
$$

where $\rho$ denotes the density of air at sea level, i.e., $1.2 \mathrm{Kg} / \mathrm{m}^{3}$, $M$ is the mass of the drone plus payload, $g$ is the gravitational force, i.e., $9.8 \mathrm{~N} / \mathrm{Kg}$, and $A$ is the rotor area. We observe that $P_{\text {drone }}$ can be assumed as a constant value since it depends only on the physical dimensions and overall weight of the drone. The expression that regulates the total power consumed by an ABS is

$$
P_{\text {consumed }}=P_{T X}+P_{\text {drone }} .
$$

Therefore, from this model, we understand that the power consumed by the ABS is entirely dependent on $P_{T X}$, whose behavior will be discussed in Sec. VIII-B. Also, in the real flights, the power consumption by the ABS will also depend on several atmospheric factors such as wind, altitude, speed of the drone, time of flight, trajectory of the drone etc, as discussed in [15].

\section{Air-To-Ground Communication Channel Model}

As given in [9], [16], the A2G channel is well-defined as probabilistic LOS and Non-LOS (NLOS) models and CloseIn reference distance model. The PL due to large scale fading and shadow fading in $\mathrm{dB}$ is defined by

$P L_{L O S}(d)[\mathrm{dB}]=20 \log _{10}\left(\frac{4 \pi d_{0}}{\lambda}\right)+10 \eta_{L O S} \log _{10}(d)+X_{\sigma, L O S}$

and

$$
\begin{aligned}
P L_{N L O S}(d)[\mathrm{dB}] & =20 \log _{10}\left(\frac{4 \pi d_{0}}{\lambda}\right) \\
& +10 \eta_{N L O S} \log _{10}(d)+X_{\sigma, N L O S}
\end{aligned}
$$

for LOA and NLOSE, respectively, where $\lambda$ is the wavelength in meters, $\eta$ is the PLE, $d$ is the link distance in meters, $d_{0}$ is the reference distance, here assumed $d_{0}=1 \mathrm{~m}$ and $X_{\sigma}$ is the log-normal random variable (Gaussian in $\mathrm{dB}$ ) with standard deviation $\sigma$ that models the large scale shadowing. The total path loss is given by

$$
P L(d)[\mathrm{dB}]=P_{L O S} \cdot P L_{L O S}(d)+\left(1-P_{L O S}\right) \cdot P L_{N L O S}(d),
$$

where $P_{L O S}$ is the probability to have a LOS link [9]. Also, for our simulations we obtain the Path Loss Exponent (PLE) from the simulation results as in [16].

For defining the small scale fading characteristics for our simulations, the channel power gain is assumed to follow a Rician distribution since in the in $\mathrm{A} 2 \mathrm{G}$ channel there is a dominant LOS component. However, this also depends on the environment chosen, as seen from the results that will be discussed in Sec. VIII.

\section{CAPACITY ANALYSIS}

Channel capacity is the key parameter that defines the performance of a communication system. It is the upper bound limit on the rate at which information can be reliably transmitter over a wireless communication channel with arbitrarily low probability of error. We assume a flat fading channel with stationary and ergodic time-varying gain $g(i)$, with $g(i) \geq 0$, and Additive White Gaussian Noise (AWGN) $n(i)$ at each time $i$. We assume that $g(i)$ is an independent and identically distributed random process distributed according to Rician probability density function in order to take into account small scale fading effects. However, the majority of propagation loss is due to large scale fading. Capacity is defined as [17]

$$
C=B \log _{2}(1+\mathrm{SNR})
$$

where $B$ is signal bandwidth and SNR is the instantaneous Signal-to-Noise Ratio (SNR) defined by

$$
\operatorname{SNR}(i)=\frac{\overline{P_{T X}}(i)}{P_{N}}=\frac{\overline{P_{T X}} g^{2}(i)}{N_{0} B}
$$

with $\overline{P_{T X}}$ denoting the average transmitted power, $P_{N}$ being the average noise power. In the above equation $N_{0}$ is the power spectral density of AWGN $n(i)$. Since $\overline{P_{T X}} / P_{N}$ is a constant, the distribution of $\operatorname{SNR}(i)$ is the same as that of $g^{2}(i)$.

\section{INTERFERENCE ANALYSIS}

Another important aspect we analyze in this paper is the impact of inter-cell interference coming from the adjacent cells. This affects the received power of a GS and, therefore, it defines the areas of the cell covered by the ABS. To analyze this, we have described the phenomenon of the interference as a function of the distance and of the PLE obtained from analyzing the data provided by the Wireless InSite simulator. We can describe the SIR as follows. Considering the ABS positioned in the center of the interfering cells and limiting the analysis to the first tier of adjacent cells, we have

$$
\mathrm{SIR}=\frac{P_{r x}}{P_{I}}=\frac{d^{-\eta}}{\sum_{i=1}^{l} r_{i}^{-\eta}}
$$

where $P_{r x}$ is the received power and $P_{I}$ is the power of the interference. In the above equation $d$ is the distance of each receiver from primary ABS, $l$ is the number of interfering ABS, and $r$ is the distance of each receiver from each interfering ABS. Here six interfering ABSs are assumed, as happens for a terrestrial scenario to compare existing cellular architectures. However, we can assume more than six interfering ABSs since the cells are moving and interference could be avoided using an appropriate trajectory planning for the ABS. The SINR can be calculated using (8) and (9) as

$$
S I N R=\frac{P_{r x}}{P_{N}+P_{I}}=\frac{\mathrm{SNR} \cdot \mathrm{SIR}}{\mathrm{SNR}+\mathrm{SIR}} .
$$

The considered interference scenario is shown in Fig. 3. Here the coverage area for each ABS is clearly shown. Actually, to have interference coverage areas should overlap but for clear understanding of the scenario, in the Figure we 


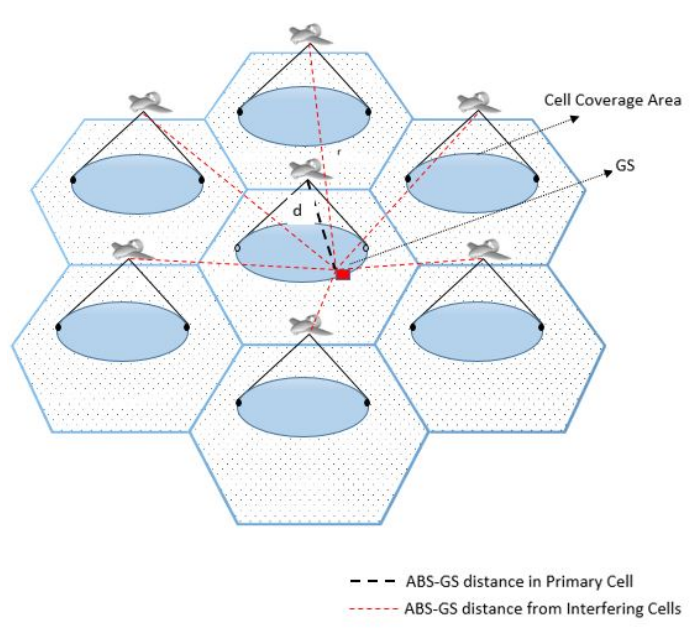

Figure 3. Interefernce Scenario from interfering ABS

prefer representing them separated in order to make them clearly distinguishable.

\section{RESULTS AND DISCUSSION}

In this Section, we plot the coverage, capacity and interference results with respect to Tx heights and Tx power. The results plotted with respect to Tx height were simulated at $18 \mathrm{dBm}$ Tx power and those plotted with respect to Tx power were simulated at $320 \mathrm{~m}$. We choose $320 \mathrm{~m}$, because it was obtained as an optimal altitude for ABS as we will see in Sec. VIII-A. Also, $18 \mathrm{dBm}$ was selected keeping in consideration the payload and dimensioning constraints of $\mathrm{UAV}$, for generating higher Tx power.

\section{A. Cell Coverage Analysis with variation in ABS height}

From the ray tracing simulation data, we obtained the received power for each $\mathrm{Rx}$ in two snapshots for each environment and plotted the percentage of cell coverage by an ABS as shown in Fig. 4. The cell coverage was obtained using (1). From Fig.4, it can be observed that maximum cell coverage increases with lesser received power threshold at the edge of the cell. In our simulation, the maximum coverage was obtained by setting the threshold to $-120 \mathrm{dBm}$. We assumed practical Received Signal Strength Indicator (RSSI) values for our simulations as obtained in practical scenario to maintain the accuracy of our results. As we increased the RSSI to $-80 \mathrm{dBm}$, the cell coverage decreases because less number of Rxs full fill the probability condition in (1). Also, it was found that the optimal altitude of ABS for maximum coverage at $18 \mathrm{dBm}$ was between $300-350 \mathrm{~m}$ for all environments. This was verified from [18], where an analytical framework was used to find ABS coverage probability for downlink user. However, with increase in Tx power, the coverage is expected to increase as seen in Sec. VIII-B. Finally, we also analyze that cell coverage is higher for Suburban environment and lowest for Urban high rise for $-120 \mathrm{dBm}$ threshold. However, we see this trend changing as the threshold increases, where Suburban has the least coverage than Urban and Urban high

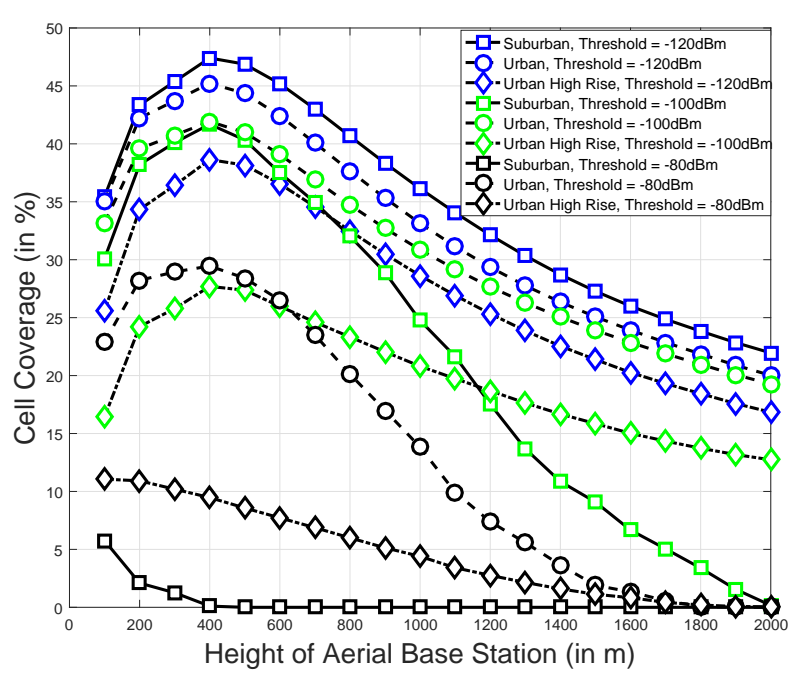

Figure 4. Coverage Analysis from an ABS at $18 \mathrm{dBm}$ Tx power for different Rx threshold

rise environments. This is because, in urban conditions, A2G channel experiences Rician fading due to the presence of LOS path. In suburban areas, a Rayleigh fading is experienced due to the presence of reflected signals that are stronger than LOS [19]. This is opposite for terrestrial communication. Therefore, as received power threshold is increased, fading changes from Rayleigh to Rician in Suburban environments and vice versa in Urban. So, a generalized approach is to use a Rician distribution where both LOS and NLOS paths are considered.

\section{B. Cell Coverage Analysis with variation in ABS Transmitted Power}

As mentioned in Sec. VIII-A, the cell coverage is expected to increase as the transmitted power of the ABS increases. This can be seen in Fig. 5. The simulations were carried for one snapshot in each environment at optimal ABS height. As seen from the simulations, the cell coverage increases linearly with $\mathrm{Tx}$ power at $-80 \mathrm{dBm}$ but becomes almost constant at $-120 \mathrm{dBm}$. This is because the Rxs receiving $-120 \mathrm{dBm}$ are already receiving the least value of received power to maintain the connectivity with the ABS. Therefore, the Rxs receiving lesser than $-120 \mathrm{dBm}$ are not present in the coverage area of the cell. Also, as expected the cell coverage is higher for Suburban environment than Urban environments, due to Rayleigh fading where multi-path and scattering effects dominate leading to constructive and destructive addition of received power with their phase and delay and therefore leading to higher received power. Finally, we also analyze from (3) and (2), that dependency of cell coverage will follow a similar behavior with respect to $P_{\text {consumed }}$.

\section{Capacity Analysis above Threshold with variation in ABS height}

For the capacity analysis, we refer (7) and (8). We set a threshold for the received power for each environment and 


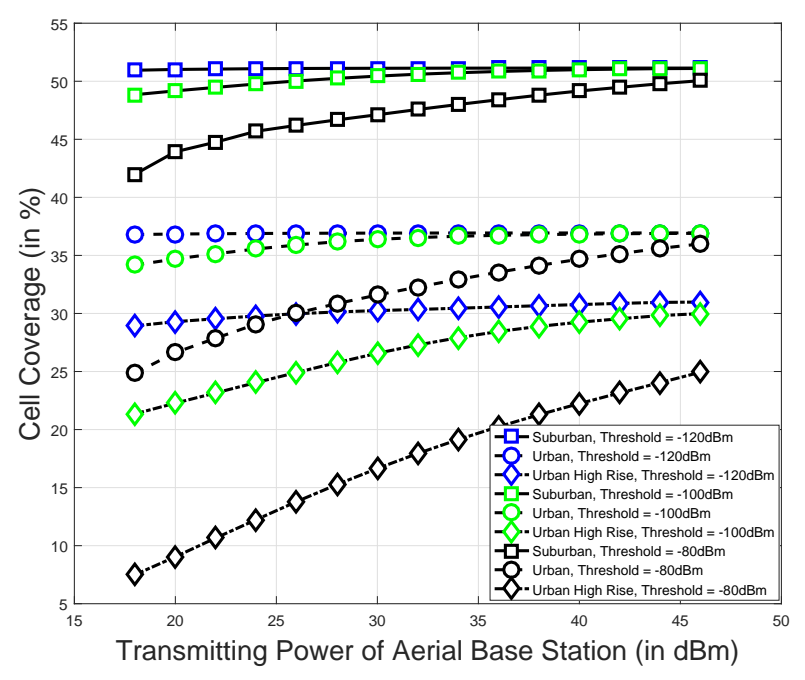

Figure 5. Coverage Analysis from an ABS at $320 \mathrm{~m}$ height with varying Tx power for different Rx threshold

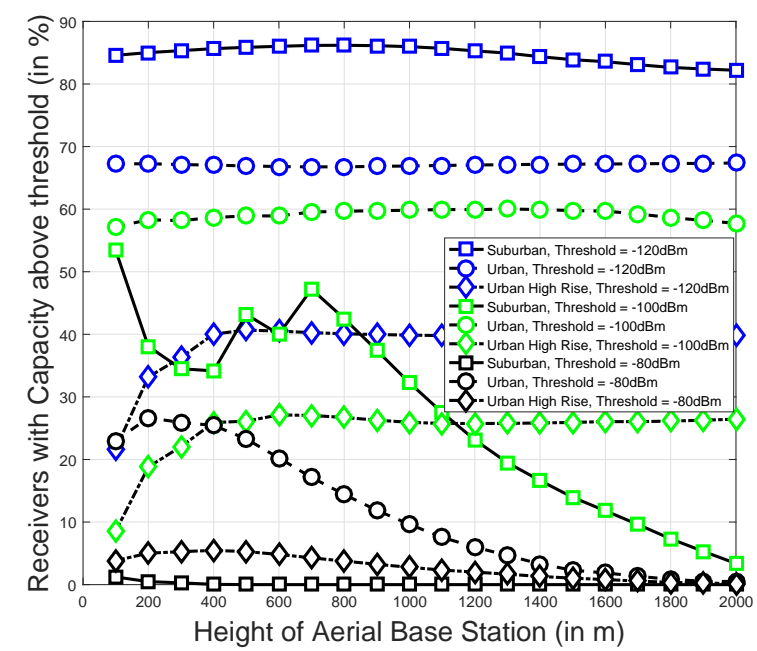

Figure 6. Capacity Analysis from an ABS at $18 \mathrm{dBm}$ Tx power for different Rx threshold

snapshot as $-120 \mathrm{dBm}$. The value was chosen considering practical RSSI in cellphones. For this threshold of received power, we obtained, the SNR threshold from (8), considering only AWGN channel and thermal noise of the receiver. Therefore, after formulating the capacity, we investigated on the percentage of Rxs, getting capacity above the threshold for different ABS heights. This result is depicted in Fig. 6. We observe that since at $-120 \mathrm{dBm}$ threshold, the SNR threshold is also minimum, so capacity of Rxs is highest. As the threshold increases, the capacity decreases. However we do not see any change in behavior for percentage of Rxs having capacity above the threshold, with respect to change in height of ABS. But we see significant drop, in Suburban scenario at $-100 \mathrm{dBm}$ and $-80 \mathrm{dBm}$. This is probably due to different fading environment than the other cases, as mentioned previously.

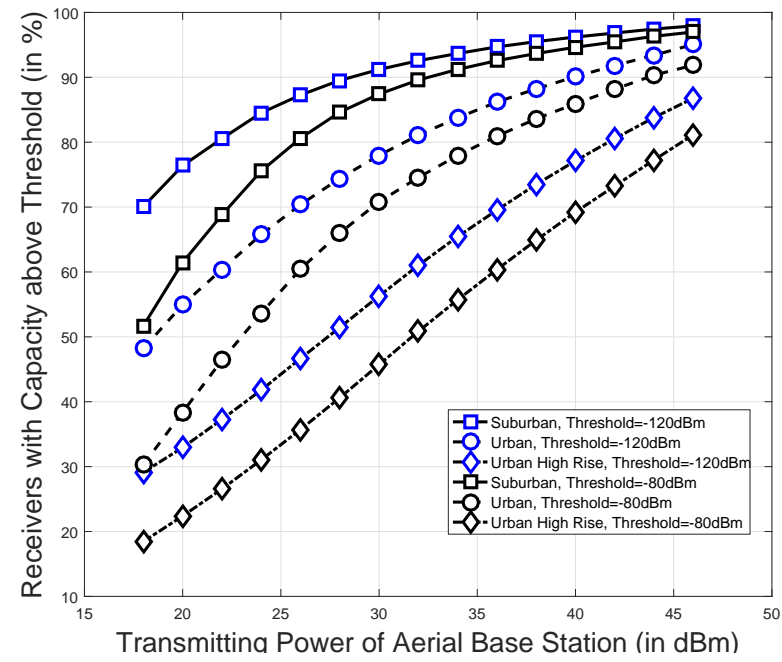

Figure 7. Capacity Analysis from an ABS at 320m height with varying Tx power for different Rx threshold

\section{Capacity Analysis above Threshold with variation in ABS Transmitted Power}

In Fig.7, we considered the cases for minimum and maximum RSSI for the simulations carried. We see that percentage of receivers with capacity above the threshold, increases with increase in transmitted power as expected, since as per (8) and (7), the SNR increase linearly with transmitter power and capacity increases logarithmically with SNR. Similarly, as we observed in Sec.VIII-C, the percentage is higher for simulation with $-120 \mathrm{dBm}$ threshold than $-80 \mathrm{dBm}$. Also, the behavior of percentage is similar at both thresholds unlike the variation due to Tx height, with Suburban having the maximum percent than Urban and Urban High rise. The reason being again due to Rayleigh fading in Suburban and Rician for Urban environments.

\section{E. SINR Analysis above Threshold with variation in ABS} Transmitted Power

Figure 8 discusses about the SINR calculated at different ABS heights. All interfering ABSs were assumed to be operating with the same parameters as the primary ABS. While considering one interfering $\mathrm{ABS}$, it was observed that percentage of Rxs with SINR above the threshold follow a constant behavior for received power threshold kept as $-120 \mathrm{dBm}$. However, for higher thresholds like -100 and $-80 \mathrm{dBm}$, the SINR tends to zero, i.e., there is high interference from one ABS. For a new set of simulations with 6 interfering $\mathrm{ABS}$, it was seen that percentage decreases rapidly for $-120 \mathrm{dBm}$, and still remains zero for higher thresholds. Also, since the simulations were carried out at $18 \mathrm{dBm} \mathrm{Tx}$ power, we recognize that it was needed to increase the $\mathrm{Tx}$ power in order to have a better SINR for each $\mathrm{Rx}$ in the scenario. 


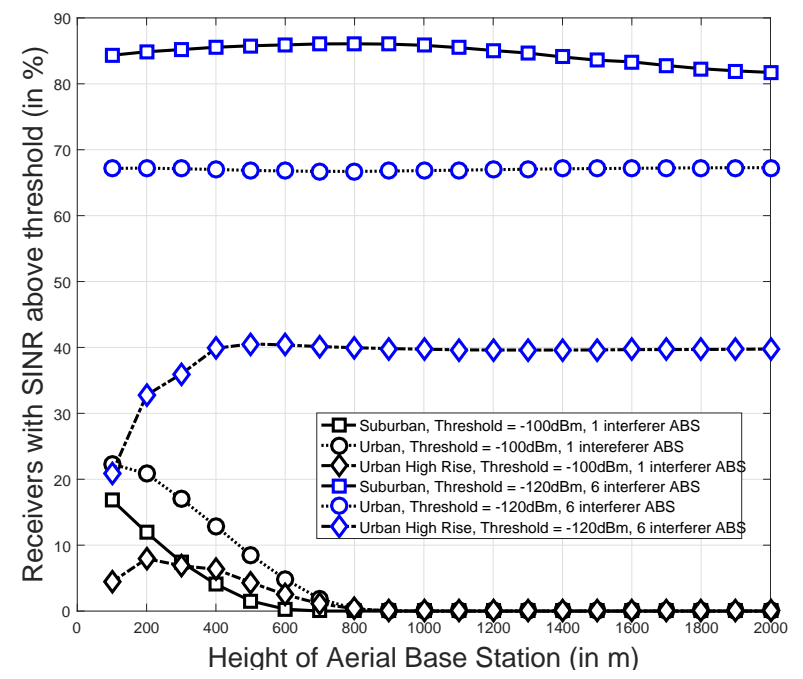

Figure 8. SINR Analysis from an ABS at 320m height with varying Tx power for different Rx threshold

\section{CONCLUSiOnS}

In this paper, analysis for Cell Coverage, Capacity and Intercell Interference from ray tracing simulation results for an Aerial Base Station (ABS) at $2.4 \mathrm{GHz}$ in different generalized environments, created following ITU-R parameters was done. From coverage results the optimal altitude for ABS was reported and a coverage dependency for power transmitted by flying base station was shown. Also, capacity and signal to interference plus noise results were presented with the percentage of receivers getting them above the thresholds, which were set keeping $-120 \mathrm{dBm},-100 \mathrm{dBm}$ and $-80 \mathrm{dBm}$ of received power, taking into account practical RSSI values.

\section{REFERENCES}

[1] M. A. Ma'sum, M. K. Arrofi, G. Jati, F. Arifin, M. N. Kurniawan, P. Mursanto, and W. Jatmiko, "Simulation of intelligent unmanned aerial vehicle (uav) for military surveillance," in 2013 International Conference on Advanced Computer Science and Information Systems (ICACSIS), Sept 2013, pp. 161-166.

[2] W. Zafar and B. M. Khan, "Flying ad-hoc networks: Technological and social implications," IEEE Technology and Society Magazine, vol. 35, no. 2, pp. 67-74, June 2016.

[3] S. Hayat, E. Yanmaz, and R. Muzaffar, "Survey on unmanned aerial vehicle networks for civil applications: A communications viewpoint,' IEEE Communications Surveys Tutorials, vol. 18, no. 4, pp. 2624-2661, Fourthquarter 2016

[4] M. Mozaffari, W. Saad, M. Bennis, and M. Debbah, "Optimal transport theory for power-efficient deployment of unmanned aerial vehicles," in Communications (ICC), 2016 IEEE International Conference on. IEEE, 2016, pp. 1-6.

[5] R. Yaliniz, A. El-Keyi, and H. Yanikomeroglu, "Efficient 3-d placement of an aerial base station in next generation cellular networks," arXiv preprint arXiv:1603.00300, 2016.

[6] E. ABSOLUTE, "Fp7 absolute project."

[7] M. M. Azari, F. Rosas, K. C. Chen, and S. Pollin, "Joint sum-rate and power gain analysis of an aerial base station," in 2016 IEEE Globecom Workshops (GC Wkshps), Dec 2016, pp. 1-6.

[8] A. AL-Hourani, S. Chandrasekharan, G. Kaandorp, W. Glenn, A. Jamalipour, and S. Kandeepan, "Coverage and rate analysis of aerial base stations [letter]," IEEE Transactions on Aerospace and Electronic Systems, vol. 52, no. 6, pp. 3077-3081, December 2016.
[9] A. Al-Hourani, S. Kandeepan, and S. Lardner, "Optimal lap altitude for maximum coverage," IEEE Wireless Communications Letters, vol. 3, no. 6, pp. 569-572, 2014.

[10] https://www.autodesk.com/products/3ds-max/overview.

[11] http://www.remcom.com/wireless-insite.

[12] P. Medeović, M. Veletić, and Ž. Blagojević, "Wireless insite software verification via analysis and comparison of simulation and measurement results," in MIPRO, 2012 Proceedings of the 35th International Convention. IEEE, 2012, pp. 776-781.

[13] F. Richter, A. J. Fehske, and G. P. Fettweis, "Energy efficiency aspects of base station deployment strategies for cellular networks," in 2009 IEEE 70th Vehicular Technology Conference Fall, Sept 2009, pp. 1-5.

[14] https://www.wired.com/2013/12/physics-of-the-amazon-prime-airdrone/.

[15] J. I. Vasquez-Gomez, C. Gomez-Castañeda, E. M. De Cote, and J. C. Herrera-Lozada, "Multirotor uav coverage planning under wind conditions," in Mechatronics, Electronics and Automotive Engineering (ICMEAE), 2016 International Conference on. IEEE, 2016, pp. 32-37.

[16] S. Sun, T. S. Rappaport, S. Rangan, T. A. Thomas, A. Ghosh, I. Z. Kovacs, I. Rodriguez, O. Koymen, A. Partyka, and J. Jarvelainen, "Propagation path loss models for $5 \mathrm{~g}$ urban micro-and macro-cellular scenarios," in Vehicular Technology Conference (VTC Spring), 2016 IEEE 83rd. IEEE, 2016, pp. 1-6.

[17] A. Goldsmith, Wireless communications. Cambridge university press, 2005.

[18] M. Mozaffari, W. Saad, M. Bennis, and M. Debbah, "Unmanned aerial vehicle with underlaid device-to-device communications: Performance and tradeoffs," IEEE Transactions on Wireless Communications, vol. 15, no. 6, pp. 3949-3963, June 2016

[19] A. Zajić, Mobile-to-mobile wireless channels. Artech House, 2012. 